\title{
Group I mGluR Agonist-Evoked Long-Term Potentiation in Hippocampal Oriens Interneurons
}

\author{
Caroline Le Duigou and Dimitri M. Kullmann \\ Department of Clinical and Experimental Epilepsy, UCL Institute of Neurology, University College London, Queen Square, London WC1N 3BG, United \\ Kingdom
}

Several subtypes of interneurons in the feedback circuit in stratum oriens of the hippocampus exhibit NMDA receptor-independent long-term potentiation (LTP) at glutamatergic synapses made by local pyramidal neurons. LTP has been reported with both "Hebbian" and "anti-Hebbian" induction protocols, where high-frequency presynaptic stimulation is paired with either postsynaptic depolarization or hyperpolarization. Do these phenomena represent distinct forms of plasticity, dependent on group I metabotropic receptors (mGluRs) and rectifying $\mathrm{Ca}^{2+}$-permeable AMPA receptors, respectively? Blockade of either mGluR1 or mGluR5 prevented anti-Hebbian LTP induction in stratum oriens interneurons in rat hippocampal slices. Exogenous activation of group I mGluRs by the selective agonist (S)-3,5-dihydroxyphenylglycine (DHPG) was unable to induce LTP on its own, and instead depressed excitatory transmission. However, when paired with postsynaptic hyperpolarization, DHPG or the group I metabotropic receptor (mGluR5)-selective agonist $(R, S)$-2chloro-5-hydroxyphenylglycine (CHPG) elicited a delayed long-lasting potentiation, which was accompanied by a decrease in pairedpulse facilitation. Anti-Hebbian LTP occluded the effect of DHPG paired with hyperpolarization, implying that the induction cascades triggered by both conjunctions of stimuli converge on common expression mechanisms.

\section{Introduction}

Several subtypes of hippocampal interneurons exhibit NMDA receptor-independent long-term potentiation (LTP) at glutamatergic synapses made by local pyramidal neurons. One induction protocol relies on 5-100 Hz presynaptic stimulation paired with postsynaptic hyperpolarization (Lamsa et al., 2007; Oren et al., 2009; Nissen et al., 2010). This phenomenon, which mainly occurs in the feedback inhibitory circuit, has been termed "anti-Hebbian" to distinguish it from associative NMDA receptor-dependent LTP in principal cells, whose induction requirements are reminiscent of Hebb's postulate (Kullmann and Lamsa, 2007). The induction mechanisms of NMDA receptor-independent LTP in the feedback circuit are incompletely understood. It occurs at synapses equipped with strongly rectifying AMPA receptors, and is prevented by selective blockers of AMPA receptors (Oren et al., 2009). Indeed, LTP dependent on $\mathrm{Ca}^{2+}$-permeable AMPA receptors has been reported in several other interneurons (Mahanty and Sah, 1998; Polepalli et al., 2010; Sambandan et al., 2010). However, a "Hebbian" form of LTP has also been reported in stratum oriens interneurons, induced by pairing theta-burst presynaptic stimulation with postsynaptic stimulation (Perez et al., 2001; Pelletier and Lacaille, 2008). This form of LTP requires the mGluR1 subtype of group I mGluRs, because it is prevented by pharmacological blockade (Perez et al., 2001) or genetic ablation

Received Dec. 1, 2010; revised Jan. 25, 2011; accepted Feb. 23, 2011.

This work was supported by the Medical Research Council, the Wellcome Trust, and the European Research Council. We are grateful to K. Lamsa, I. Oren, and E. Nicholson for helpful comments.

Correspondence should be addressed to Dimitri M. Kullmann at the above address. E-mail: D.Kullmann@ ion.ucl.ac.uk.

DOI:10.1523/JNEUROSCI.6265-10.2011

Copyright $\odot 2011$ the authors $\quad 0270-6474 / 11 / 315777-05 \$ 15.00 / 0$
(Lapointe et al., 2004) of this receptor. mGluR1-dependent LTP has also been reported at synapses made by mossy fibers on interneurons in stratum lacunosum/moleculare (Galván et al., 2008). Interestingly, at synapses made by pyramidal neurons on fast-spiking interneurons in layer $2 / 3$ of the visual cortex, LTP depends not on mGluR1 but on the other group I mGluR subtype, mGluR5 (Sarihi et al., 2008).

Although many of the studies listed above have examined different synapses and are therefore not directly comparable (see also Cowan et al., 1998; Alle et al., 2001; Laezza and Dingledine, 2004; Pelkey et al., 2005), the reports of Hebbian and anti-Hebbian NMDA receptor-independent LTP in stratum oriens have focused on overlapping, if not congruent, populations of interneurons innervated by local pyramidal neurons (Perez et al., 2001; Lapointe et al., 2004; Lamsa et al., 2007; Oren et al., 2009; Croce et al., 2010; Nissen et al., 2010), which include oriens-lacunosum/moleculare (O-LM) cells (Lacaille et al., 1987; Blasco-Ibáñez and Freund, 1995). Are the LTP dependent on $\mathrm{Ca}^{2+}$-permeable AMPA receptors and the LTP dependent on group I mGluRs two sides of the same coin? Both forms of LTP appear to be expressed presynaptically, as witnessed by changes in failure rates, trial-to-trial variability, pairedpulse facilitation, and sensitivity to use-dependent AMPA receptor blockers (Perez et al., 2001; Lamsa et al., 2007; Croce et al., 2010). Indeed, because LTP has been studied with either whole-cell (Perez et al., 2001; Lapointe et al., 2004; Croce et al., 2010), cell-attached (Croce et al., 2010), or perforated-patch pipettes (Lamsa et al., 2007; Oren et al., 2009; Nissen et al., 2010), subtle differences in recording methods could potentially reconcile the different reports.

Here, we show that mGluR1 and mGluR5 are necessary for induction of anti-Hebbian LTP. Although exogenous activation of group I mGluRs with (S)-3,5-dihydroxyphenylglycine (DHPG) 
fails to elicit LTP on its own, when paired with postsynaptic hyperpolarization, a slowly evolving but long-lasting potentiation is evoked. This is associated with a decrease in paired-pulse facilitation and is occluded by anti-Hebbian LTP. The results imply that a group I mGluR-dependent cascade contributes, together with $\mathrm{Ca}^{2+}$-permeable AMPA receptors, to the induction of antiHebbian LTP.

\section{Materials and Methods}

Hippocampal slices. All procedures followed the Animals (Scientific Procedures) Act, 1986. Transverse $350 \mu \mathrm{m}$ hippocampal slices were prepared from postnatal day 20-24 male Sprague Dawley rats. Animals were decapitated under terminal pentobarbital anesthesia $(140 \mathrm{mg} / \mathrm{kg})$. Slices were cut with a vibratome (VT1000S, Leica) in an ice-cold solution containing (in mM): 70 sucrose, $80 \mathrm{NaCl}, 2.5 \mathrm{KCl}, 1.25 \mathrm{NaH}_{2} \mathrm{PO}_{4}, 25$ $\mathrm{NaHCO}_{3}, 7 \mathrm{MgCl}_{2}, 0.5 \mathrm{CaCl}_{2}, 25$ glucose ( $\mathrm{pH} 7.3,315 \mathrm{mOsm}$ ), bubbled with $95 \% \mathrm{O}_{2} / 5 \% \mathrm{CO}_{2}$. Slices were allowed to recover at room temperature in an interface chamber, in carbogen-bubbled $\mathrm{NaCl}(119 \mathrm{mM}), \mathrm{KCl}$ (2.5 mM), $\mathrm{NaHCO}_{3}(26 \mathrm{~mm}), \mathrm{NaH}_{2} \mathrm{PO}_{4}(1 \mathrm{~mm}), \mathrm{MgCl}_{2}$ (3 mM), $\mathrm{CaCl}_{2}(2$ $\mathrm{mm})$, glucose (15 mM) ( $\mathrm{pH} 7.3,297 \mathrm{mOsm})$, and then transferred to the recording chamber of an upright microscope (BX50WI, Olympus). The perfusion solution $\left(3 \mathrm{ml} / \mathrm{min}, 30-32^{\circ} \mathrm{C}\right)$ was the same as the storage solution except that $\mathrm{CaCl}_{2}$ was increased to $2.5 \mathrm{~mm}$, and $\mathrm{Mg}^{2+}$ was reduced to 1.3 $\mathrm{mm}$ and was supplemented with picrotoxin $(100 \mu \mathrm{M})$ and 3-[[(3,4dichlorophenyl)methyl]amino]propyl]diethoxymethyl)phosphinic acid (CGP 52432) (1 $\mu \mathrm{M})$ to block GABA receptors, and with DL-2-amino-5phosphonovalerate (DL-APV) $(50 \mu \mathrm{M})$ to block NMDA receptors. A cut was made between CA3 and CA1.

Electrophysiology and analysis. Interneurons in stratum oriens of the CA1 subfield with dendrites running parallel to stratum pyramidale were patchclamped under infrared differential interference contrast microscopy, using a Multiclamp 700B amplifier (Molecular Devices). Records were filtered at 5 $\mathrm{kHz}$, digitized at $10 \mathrm{kHz}$, and recorded using programs written in Labview (National Instruments). For perforated-patch current-clamp recordings, gramicidin $\left(100 \mathrm{mg} \cdot \mathrm{ml}^{-1}\right.$; Sigma-Aldrich), prepared daily in DMSO, was added $(1: 1000)$ to a pipette solution containing (in $\mathrm{mM}$ ): 130 K-gluconate, $8 \mathrm{NaCl}, 20$ HEPES, 0.2 EGTA, and 5 QX-314 $\mathrm{Br}$ [2(triethylamino)- $\mathrm{N}$-(2,6-dimethylphenyl)acetamine bromide ( $\mathrm{pH} \mathrm{7.2,}$ $295 \mathrm{mOsm}$ )]. The pipette tip was filled with gramicidin-free solution. Recordings were started when the series resistance was $<150 \mathrm{M} \Omega$, and the experiment was discontinued if depolarizing pulses failed to evoke action potentials. The membrane potential was maintained between -75 and $-65 \mathrm{mV}$ with current injections where necessary. For whole-cell current-clamp recordings, the pipette solution contained (in mM) 117.5 K-gluconate, $17.5 \mathrm{KCl}, 10 \mathrm{CsOH}$ HEPES, 0.2 EGTA, $8 \mathrm{NaCl}, 2 \mathrm{MgATP}$, $0.3 \mathrm{Na}_{3} \mathrm{GTP}, 5 \mathrm{QX}-314 \mathrm{Br}$ (pH 7.2, $296 \mathrm{mOsm}$ ). The pipette resistance was 4-5 M $\Omega$. Neurons were voltage-clamped at $-60 \mathrm{mV}$. Cells were rejected if either the series resistance or the holding current varied by $>25 \%$.

Two bipolar stainless steel electrodes were positioned in the alveus/ stratum oriens border on either side of the interneuron (distance 100$500 \mu \mathrm{m}$ ), to stimulate axon collaterals of pyramidal neurons. Either single or paired stimuli (50-100 $\mu$ s duration, $50 \mathrm{~ms}$ interpulse interval; Digitimer) were alternately delivered via each electrode, with a $15 \mathrm{~s}$ duty cycle. Paired-pulse ratios (PPRs) were calculated from averages of 20 cycles. LTP was induced in one pathway by stimulating at $100 \mathrm{~Hz}$ for $1 \mathrm{~s}$, twice with a $20 \mathrm{~s}$ interval. The postsynaptic cell was voltage-clamped between -90 and $-100 \mathrm{mV}$ during each train to prevent it from spiking.

To control for nonspecific drift in recording conditions, we expressed the pathway-specific potentiation as $100 \times\left(\mathrm{EPSP}_{\text {test }} / \mathrm{EPSP}_{\mathrm{control}}-1\right) \%$, where the EPSP initial slopes in test and control pathways (EPSP test and $\mathrm{EPSP}_{\text {control }}$, respectively) were first normalized by their average values before pairing. LTP was estimated from a $5 \mathrm{~min}$ period between 20 and 25 min after induction. Data were analyzed with Student's paired $t$ test, and significance was taken as $p<0.05$.

Drugs. The following drugs were obtained from Tocris Bioscience: CGP 52432, DL-APV, DHPG, LY 367385 [( S)-(+)- $\alpha$-amino-4-carboxy-2methylbenzeneacetic acid], and MPEP [2-methyl-6-(phenylethynyl) pyridine hydrochloride]. Picrotoxin was obtained from Sigma. QX314 Br and CHPG were obtained from Ascent Scientific.

\section{Results}

\section{Both mGluR1 and mGluR5 contribute to anti-Hebbian LTP induction}

We focused on interneurons with dendrites oriented parallel to stratum pyramidale, most of which correspond to O-LM cells. These interneurons abundantly express an isoform of mGluR1 (van Hooft et al., 2000; Ferraguti et al., 2004), and, together with parvalbumin-positive basket and axoaxonic cells, exhibit antiHebbian LTP (Lamsa et al., 2007; Oren et al., 2009; Nissen et al., 2010). Because this form of LTP is rapidly disrupted with wholecell recordings (Lamsa et al., 2007), we used the perforated-patch method throughout this study unless otherwise indicated.

Pairing high-frequency stimulation of one pathway $(100 \mathrm{~Hz}$, $1 \mathrm{~s}$, twice) with postsynaptic hyperpolarization elicited $54 \pm 10 \%$ potentiation, measured at $20 \mathrm{~min}$, in 40 interneurons (Fig. $1 \mathrm{~A}$ ). When applied in the continued presence of the mGluR1 blocker LY $367385(100 \mu \mathrm{M})$, the same pairing protocol only induced post-tetanic potentiation, which rapidly decayed to baseline. However, the paired pathway subsequently diverged from the control pathway, such that, after $20 \mathrm{~min}$, the EPSP slope reached a maximum $35 \pm 13 \%$ increase relative to the control pathway $(n=10, p=0.02$; Fig. $1 B)$. We also interleaved experiments in which the pairing was applied in the presence of the mGluR5 blocker MPEP $(25 \mu \mathrm{M})$. This also failed to elicit LTP: the mean EPSP slope after 20 min was $88 \pm 7 \%$ of baseline $(n=9)$, and was not significantly different from the control pathway $(p=0.33$; Fig. 1C).

Although these results suggest that both mGluR1 and mGluR5 are involved in anti-Hebbian LTP induction, a potential pitfall is that it is not possible to know whether LTP would have been induced without the blockers. We took advantage of the finding that sequentially pairing two homologous pathways results in the same pattern of plasticity at each population of synapses (Lamsa et al., 2005, 2007). We first asked whether the pathway-specific potentiation was sustained after pairing in the absence of mGluR blockers. In 10 interneurons showing LTP, we monitored the test and control pathways for up to $60 \mathrm{~min}$ after pairing. The average potentiation thus measured in the test pathway was $60 \pm 14 \%, 40$ min post-pairing $(n=5, p<0.001$; Fig. $2 A)$. We next verified that sequential pairing of two pathways elicited pathway-specific LTP. In 7 of 7 cases in which the first pairing elicited LTP, pairing the second pathway also resulted in a pathway-specific potentiation, albeit with a smaller magnitude (measured 10 min after pairing, first pathway: $54 \pm 21 \%, p<$ 0.01 ; second pathway: $28 \pm 9 \%, p<0.01$; Fig. $2 B$ ). (Attention was restricted to the first $10 \mathrm{~min}$ after pairing, because it proved difficult to maintain a stable recording for over an hour.)

We applied the sequential pairing protocol with either LY 367385 or MPEP applied after LTP was elicited in the first pathway. Pairing the second pathway in the presence of LY 367385 failed to evoke a significant potentiation (relative potentiation $10 \pm 10 \%, p=0.14, n=6$; Fig. $2 C$ ). We repeated the experiment with bath application of MPEP after the first pairing. The pairing protocol delivered to the second pathway again failed to elicit a significant potentiation $(12 \pm 6 \%, p=0.09, n=6$; Fig. $2 D)$. These results confirm that preventing either mGluR1 or mGluR5 receptor activation blocks anti-Hebbian LTP induction, at least over the first $10 \mathrm{~min}$. 
A
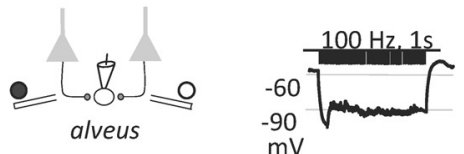

HFS
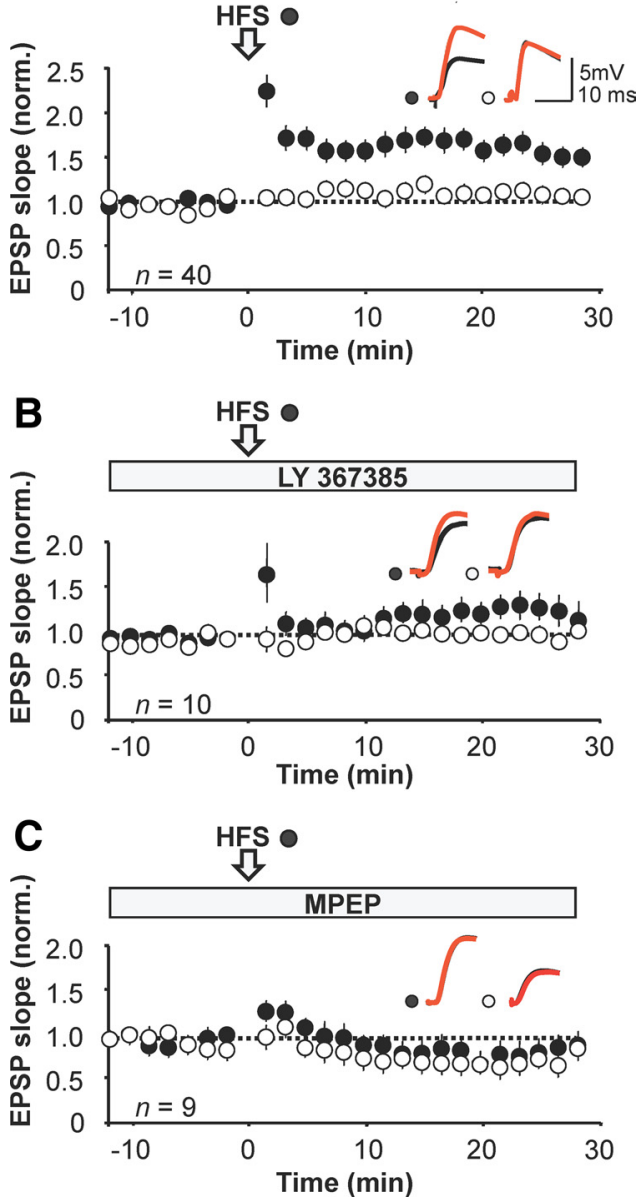

Figure 1. Blockade of either mGluR1 or mGluR5 prevents anti-Hebbian LTP induction. $A$, Baseline-normalized EPSP slope, showing the effect of pairing presynaptic highfrequency stimulation (HFS) of one pathway (filled symbols) with postsynaptic hyperpolarization (open arrow). Open symbols, Control pathway. Error bars show SEM. Top, Schematic showing the arrangement of stimulating and recording electrodes and the LTP induction protocol. Inset, Representative sample traces from a single neuron before (black) and after (red) pairing in the two pathways. $\boldsymbol{B}$, LTP protocol repeated during perfusion of the selective mGluR1 blocker LY $367385(100 \mu \mathrm{M})$. C, LTP protocol repeated during perfusion of the mGluR5 blocker MPEP $(25 \mu \mathrm{M})$.

\section{Biphasic modulation of EPSPs by group I mGluRs}

Is group I mGluR activation sufficient to induce LTP on its own? We applied the group I mGluR agonist DHPG and monitored the EPSP initial slope in perforated-patch mode. Direct current injection was used to keep the membrane potential within $5 \mathrm{mV}$ of baseline. DHPG ( $5 \mu \mathrm{M}$, applied for $10 \mathrm{~min})$ reversibly depressed EPSPs to $77 \pm 6 \%$ of baseline $(n=18, p<0.05$; Fig. $3 A)$. The depression was accompanied by a decrease in $1 / \mathrm{CV}^{2}$ and a $27 \pm 6 \%$ increase in PPR $(p=0.001)$ (data not shown). Higher concentrations of DHPG led to larger and more prolonged depression when recording either in perforated-patch or in whole-cell mode (Le Duigou et al., 2011).

We asked whether the DHPG-evoked depression could be converted to a potentiation by pairing application of the mGluR agonist with postsynaptic hyperpolarization to between $-90 \mathrm{mV}$ and $-100 \mathrm{mV}$ (similar to the LTP induction protocol) for 10 min. Pairing DHPG with hyperpolarization led to a slow increase
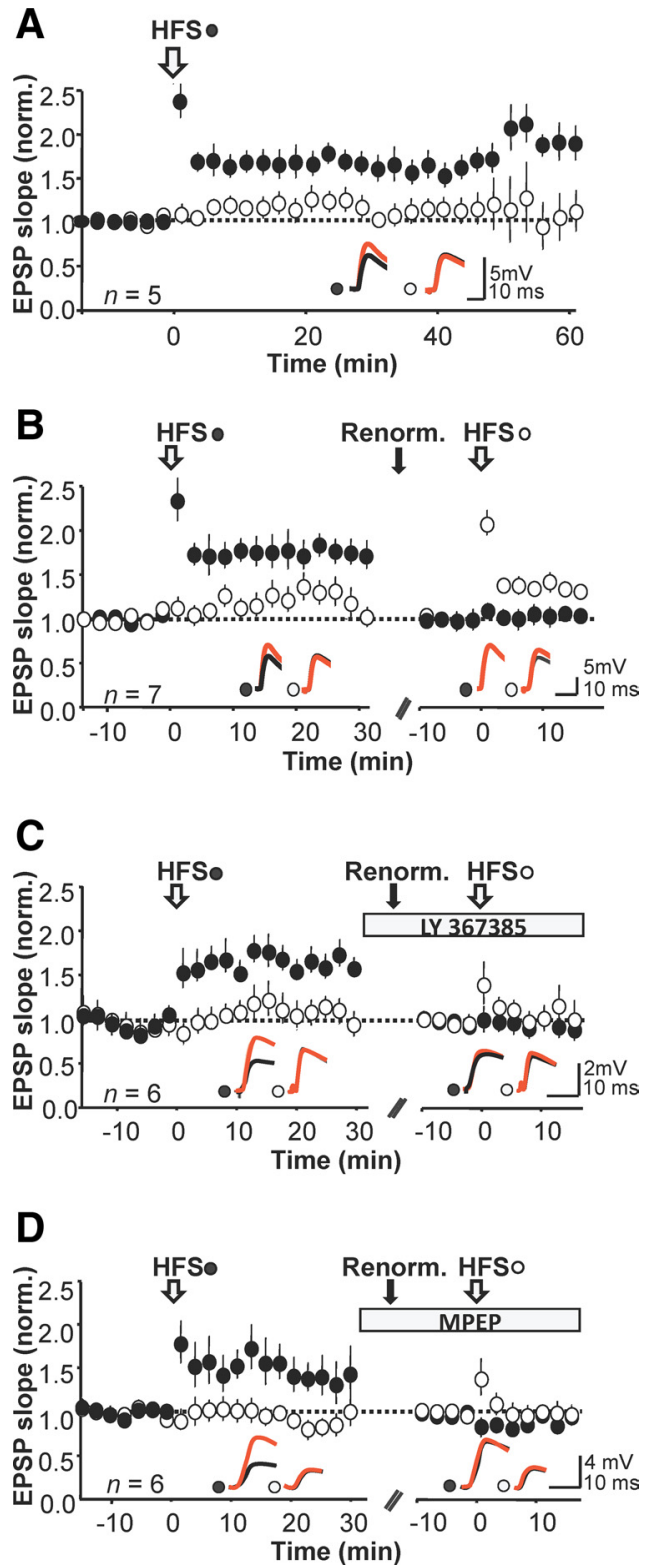

Figure 2. Consecutive pairing of two pathways reveals roles of mGluR1 and mGluR5. $\boldsymbol{A}$, Anti-Hebbian LTP persists for at least $60 \mathrm{~min}$. EPSP slope (mean \pm SEM) normalized to average baseline amplitude before induction of LTP $(n=5)$. Insets, Representative sample traces from a single neuron before (black) and after (red) pairing. $\boldsymbol{B}$, Potentiation could be induced with an anti-Hebbian protocol in the second pathway. LTP was first induced in one pathway (filled symbols), and the same protocol was subsequently applied to the other pathway (open symbols). The EPSP slopes were renormalized before the second pairing $(n=7)$. C, Blocking mGluR1 with LY 367385 prevented potentiation when washed in before applying an antiHebbian protocol to the second pathway. $\boldsymbol{D}$, Data plotted in the same way in a separate set of experiments in which mGluR5 receptors were blocked with MPEP before the second pairing.

in EPSP slope after DHPG washout in 10 of 14 interneurons (Fig. $3 B)$. On average the EPSP slope increased to $155 \pm 19 \%$ of baseline $(n=14, p<0.01)$. The delayed potentiation did not require synaptic activity during the DHPG application, because it was no smaller in a pathway whose stimulation was interrupted and then resumed after washout (stimulated pathway: $41 \pm 19 \%$ increase, $p<0.01$ relative to baseline; unstimulated pathway: $39 \pm 22 \%$ increase, $p<0.01 ; n=10$; between-pathway comparison, NS; Fig. $3 C$ ). However, we cannot rule out the possibility that spontaneous glutamate release occurred during the application of DHPG and hyperpolarization. 


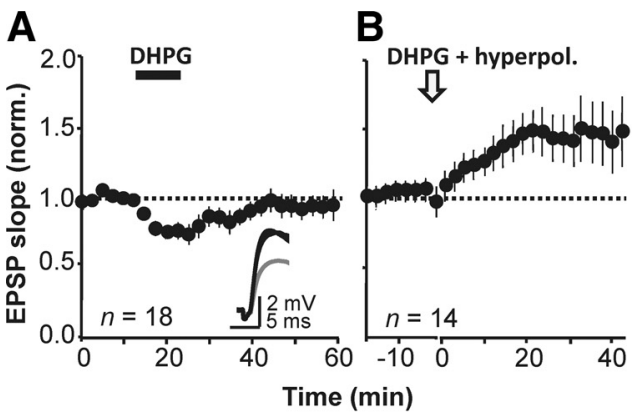

C

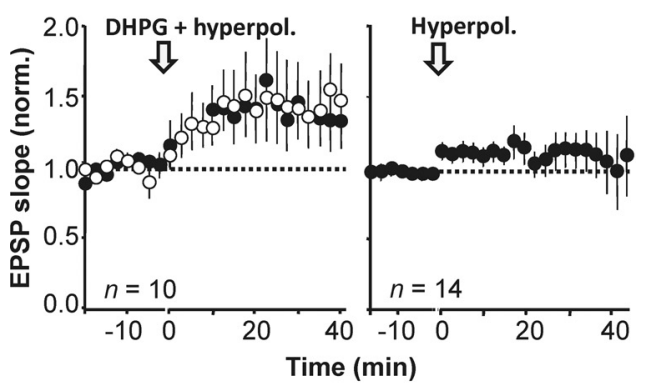

E

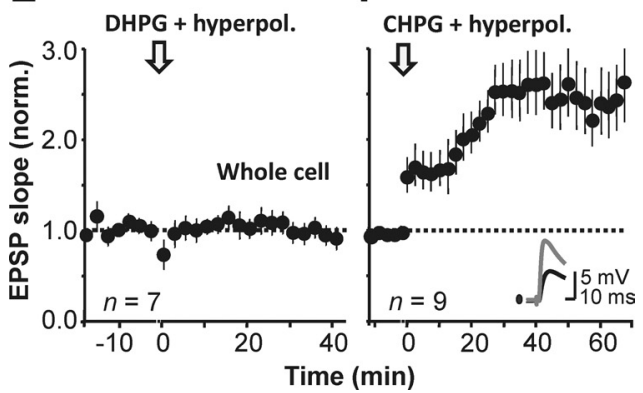

Figure 3. Bidirectional modulation of EPSPs by group I mGluRs. $A$, The group I mGluR agonist DHPG $(5 \mu \mathrm{M})$ reversibly depressed monosynaptic EPSPs in CA1 stratum oriens interneurons. Inset, Representative sample traces before, during (gray), and after DHPG application (averages of 20 sweeps). B, DHPG evoked a delayed potentiation when paired with hyperpolarization. , Interrupting stimulation of one pathway (open symbols) did not prevent the delayed potentiation induced by DHPG application together with hyperpolarization. $\boldsymbol{D}$, Hyperpolarization alone had no long-lasting effect on transmission. E, DHPG paired with hyperpolarization had no effect on transmission when recording in whole-cell mode. $\boldsymbol{F}$, The selective mGluR5 agonist CHPG (500 $\mu \mathrm{M}$ ) induced a potentiation when paired with hyperpolarization (perforated patch). Inset, Representative traces before (black) and after (gray) CHPG .

In contrast, hyperpolarization delivered on its own without DHPG application was ineffective (EPSP slope $113 \pm 7 \%$ of baseline, $p=0.19, n=14$; Fig. $3 D$ ). DHPG application also failed to evoke a robust potentiation when the recording was performed in whole-cell mode ( $97 \pm 8 \%, n=7$; Fig. $3 E$ ) even if paired with hyperpolarization, consistent with previous evidence that LTP induction in interneurons is highly sensitive to dialysis of the cytoplasm (Lamsa et al., 2005, 2007).

\section{Exogenous activation of group I mGluRs with} hyperpolarization occludes LTP

Although, as pointed out above, activation of $\mathrm{Ca}^{2+}$-permeable AMPA receptors was not completely prevented during DHPG application, an alternative explanation for the interaction of group I mGluR activation with hyperpolarization is that this triggers $\mathrm{Ca}^{2+}$ influx via voltage-gated $\mathrm{Ca}^{2+}$ or TRP channels, which are differentially coupled to mGluR1 and mGluR5 (Topolnik et

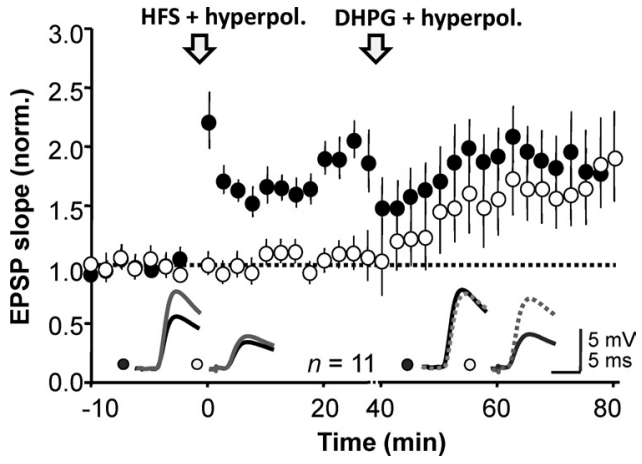

Figure 4. Anti-Hebbian LTP occludes the potentiation induced by DHPG paired with hyperpolarization. Anti-Hebbian LTP was induced in one pathway (filled symbols) and DHPG perfusion was subsequently paired with a hyperpolarization, leading to delayed potentiation that was restricted to the control pathway (open symbols). Inset, Representative sample traces before (black) and after (gray) LTP induction, and before and after DHPG application (dashed).

al., 2006, 2009). We asked whether selective activation of mGluR5 could trigger the same potentiation. The specific mGluR5 agonist CHPG $(500 \mu \mathrm{M})$ induced a delayed potentiation of $151 \pm 7 \%$ when paired with postsynaptic hyperpolarization $(p \ll 0.01$, $n=9$; Fig. $3 F$ ), which was again associated with a decrease in PPR (data not shown).

Finally, we looked for occlusion between the potentiation evoked by hyperpolarization with mGluR activation and anti-Hebbian LTP. We induced LTP by pairing high-frequency stimulation with hyperpolarization in one pathway (average potentiation $74 \pm 3 \%, n=11$, $p \ll 0.01$; Fig. 4) and then applied DHPG $(5 \mu \mathrm{M})$ together with hyperpolarization. DHPG with hyperpolarization induced a $70 \pm 3 \%$ increase in the naive pathway ( $p \ll 0.01$ ) but no change in the previously potentiated pathway. Both forms of potentiation were associated with similar changes in PPR (data not shown). Occlusion of the DHPG effect by prior induction of anti-Hebbian LTP thus argues that they converge on a common expression mechanism.

\section{Discussion}

The present results argue that both mGluR1 and mGluR5 contribute to the induction of anti-Hebbian LTP at excitatory synapses on interneurons in stratum oriens. These receptors are abundantly expressed in many neurons, and tend to be located in the postsynaptic membrane, in a perisynaptic annulus surrounding glutamatergic synapses (Lujan et al., 1996). They are also enriched in several types of interneurons (van Hooft et al., 2000; Ferraguti et al., 2004). Although they are implicated in long-term depression at synapses made by Schaffer collaterals on several targets (Anwyl, 2009) including interneurons in stratum radiatum (Gibson et al., 2008), several previous studies show that they contribute to LTP in interneurons in stratum oriens (Perez et al., 2001; Lapointe et al., 2004). Demonstrating that an induction cascade is sufficient to lead to any form of long-term synaptic plasticity is notoriously difficult (Sanes and Lichtman, 1999). Nevertheless, the involvement of group I mGluRs in LTP is reinforced by the finding that exogenous application can lead to a delayed potentiation (Le Vasseur et al., 2008). In that study, depolarization was necessary to uncover the potentiation. The present study differs in that perforated-patch recordings were used to minimize cytoplasmic disruption. With this recording method, LTP occurs at synapses with strongly rectifying (and by implication, $\mathrm{Ca}^{2+}$-permeable) AMPA receptors (Lamsa et al., 2007; Nissen et al., 2010), and reversible blockade of these receptors 
prevents its induction (Oren et al., 2009). This evidence taken in isolation implies an instructive role for $\mathrm{Ca}^{2+}$ influx via AMPA receptors (Mahanty and Sah, 1998). However, we now show that exogenous activation of group I mGluRs with DHPG can itself induce a delayed potentiation, but only if paired with hyperpolarization. Importantly, this is occluded by prior induction of anti-Hebbian LTP, implying that the two phenomena indeed converge on a common cascade.

mGluR1 and mGluR5 exhibit distinct interactions with intracellular stores and transient receptor potential channels (Topolnik et al., 2006). Although selective activation of mGluR5 by pairing CHPG application with hyperpolarization was sufficient to evoke a long-lasting potentiation, it is difficult to argue that this receptor is preferentially linked to LTP. Indeed, we found that blockade of either mGluR1 or mGluR5 was sufficient to prevent anti-Hebbian LTP induction. A tentative model that reconciles these findings is that all three essential components $\left(\mathrm{Ca}^{2+}\right.$-permeable AMPA receptors, mGluR1, and mGluR5) contribute cumulatively to generate a local postsynaptic signal required to induce LTP. This signal may be a local increase in $\mathrm{Ca}^{2+}$, and with exogenous activation of group I mGluRs may even obviate the requirement for AMPA receptors, explaining why DHPG application with depolarization yielded a potentiation that did not differ between the stimulated and unstimulated pathways. However, when synaptic stimulation is paired with hyperpolarization, removal of any one of these components may render this signal insufficient for LTP induction and may instead uncover a depression of transmission. Indeed, in a previous study using an anti-Hebbian induction protocol, we observed a small but significant depression of the conditioned pathway when AMPA receptors were temporarily blocked (Oren et al., 2009).

\section{References}

Alle H, Jonas P, Geiger JR (2001) PTP and LTP at a hippocampal mossy fiber-interneuron synapse. Proc Natl Acad Sci U S A 98:14708-14713.

Anwyl R (2009) Metabotropic glutamate receptor-dependent long-term potentiation. Neuropharmacology 56:735-740.

Blasco-Ibáñez JM, Freund TF (1995) Synaptic input of horizontal interneurons in stratum oriens of the hippocampal CA1 subfield: structural basis of feed-back activation. Eur J Neurosci 7:2170-2180.

Cowan AI, Stricker C, Reece LJ, Redman SJ (1998) Long-term plasticity at excitatory synapses on aspinous interneurons in area CA1 lacks synaptic specificity. J Neurophysiol 79:13-20.

Croce A, Pelletier JG, Tartas M, Lacaille JC (2010) Afferent-specific properties of interneuron synapses underlie selective long-term regulation of feedback inhibitory circuits in CAl hippocampus. J Physiol 588: 2091-2107.

Ferraguti F, Cobden P, Pollard M, Cope D, Shigemoto R, Watanabe M, Somogyi P (2004) Immunolocalization of metabotropic glutamate receptor 1alpha (mGluR1alpha) in distinct classes of interneuron in the CA1 region of the rat hippocampus. Hippocampus 14:193-215.

Galván EJ, Calixto E, Barrionuevo G (2008) Bidirectional Hebbian plasticity at hippocampal mossy fiber synapses on CA3 interneurons. J Neurosci 28:14042-14055.

Gibson HE, Edwards JG, Page RS, Van Hook MJ, Kauer JA (2008) TRPV1 channels mediate long-term depression at synapses on hippocampal interneurons. Neuron 57:746-759.

Kullmann DM, Lamsa KP (2007) Long-term synaptic plasticity in hippocampal interneurons. Nat Rev Neurosci 8:687-699.

Lacaille JC, Mueller AL, Kunkel DD, Schwartzkroin PA (1987) Local circuit interactions between oriens/alveus interneurons and CA1 pyramidal cells in hippocampal slices: electrophysiology and morphology. J Neurosci 7:1979-1993.

Laezza F, Dingledine R (2004) Voltage-controlled plasticity at GluR2deficient synapses onto hippocampal interneurons. J Neurophysiol 92: 3575-3581.

Lamsa K, Heeroma JH, Kullmann DM (2005) Hebbian LTP in feed-forward inhibitory interneurons and the temporal fidelity of input discrimination. Nat Neurosci 8:916-924.

Lamsa KP, Heeroma JH, Somogyi P, Rusakov DA, Kullmann DM (2007) Anti-Hebbian long-term potentiation in the hippocampal feedback inhibitory circuit. Science 315:1262-1266.

Lapointe V, Morin F, Ratté S, Croce A, Conquet F, Lacaille JC (2004) Synapsespecific mGluR1-dependent long-term potentiation in interneurones regulates mouse hippocampal inhibition. J Physiol 555:125-135.

Le Duigou C, Holden T, Kullmann DM (2011) Short- and long-term depression at glutamatergic synapses on hippocampal interneurons by group I mGluR activation. Neuropharmacology 60:748-756.

Le Vasseur M, Ran I, Lacaille JC (2008) Selective induction of metabotropic glutamate receptor 1 - and metabotropic glutamate receptor 5-dependent chemical long-term potentiation at oriens/alveus interneuron synapses of mouse hippocampus. Neuroscience 151:28-42.

Lujan R, Nusser Z, Roberts JD, Shigemoto R, Somogyi P (1996) Perisynaptic location of metabotropic glutamate receptors mGluR1 and mGluR5 on dendrites and dendritic spines in the rat hippocampus. Eur. J Neurosci 8:1488-1500.

Mahanty NK, Sah P (1998) Calcium-permeable AMPA receptors mediate long-term potentiation in interneurons in the amygdala. Nature 394:683-687.

Nissen W, Szabo A, Somogyi J, Somogyi P, Lamsa KP (2010) Cell typespecific long-term plasticity at glutamatergic synapses onto hippocampal interneurons expressing either parvalbumin or CB1 cannabinoid receptor. J Neurosci 30:1337-1347.

Oren I, Nissen W, Kullmann DM, Somogyi P, Lamsa KP (2009) Role of ionotropic glutamate receptors in long-term potentiation in rat hippocampal CA1 oriens-lacunosum moleculare interneurons. J Neurosci 29:939-950.

Pelkey KA, Lavezzari G, Racca C, Roche KW, McBain CJ (2005) mGluR7 is a metaplastic switch controlling bidirectional plasticity of feedforward inhibition. Neuron 46:89-102.

Pelletier JG, Lacaille JC (2008) Long-term synaptic plasticity in hippocampal feedback inhibitory networks. Prog Brain Res 169:241-250.

Perez Y, Morin F, Lacaille JC (2001) A hebbian form of long-term potentiation dependent on mGluRla in hippocampal inhibitory interneurons. Proc Natl Acad Sci U S A 98:9401-9406.

Polepalli JS, Sullivan RK, Yanagawa Y, Sah P (2010) A specific class of interneuron mediates inhibitory plasticity in the lateral amygdala. J Neurosci 30:14619-14629.

Sambandan S, Sauer JF, Vida I, Bartos M (2010) Associative plasticity at excitatory synapses facilitates recruitment of fast-spiking interneurons in the dentate gyrus. J Neurosci 30:11826-11837.

Sanes JR, Lichtman JW (1999) Can molecules explain long-term potentiation? Nat Neurosci 2:597-604.

Sarihi A, Jiang B, Komaki A, Sohya K, Yanagawa Y, Tsumoto T (2008) Metabotropic glutamate receptor type 5-dependent long-term potentiation of excitatory synapses on fast-spiking GABAergic neurons in mouse visual cortex. J Neurosci 28:1224-1235.

Topolnik L, Azzi M, Morin F, Kougioumoutzakis A, Lacaille JC (2006) mGluR1/5 subtype-specific calcium signalling and induction of longterm potentiation in rat hippocampal oriens/alveus interneurones. J Physiol 575:115-131.

Topolnik L, Chamberland S, Pelletier JG, Ran I, Lacaille JC (2009) Activitydependent compartmentalized regulation of dendritic $\mathrm{Ca}^{2+}$ signaling in hippocampal interneurons. J Neurosci 29:4658-4663.

van Hooft JA, Giuffrida R, Blatow M, Monyer H (2000) Differential expression of group I metabotropic glutamate receptors in functionally distinct hippocampal interneurons. J Neurosci 20:3544-3551. 